\title{
Unexpected cross-species contamination in genome sequencing projects
}

The raw data from a genome sequencing project sometimes contains DNA from contaminating organisms, which may be introduced during sample collection or sequence preparation. In some instances, these contaminants remain in the sequence even after assembly and deposition of the genome into public databases. As a result, searches of these databases may yield erroneous and confusing results. We used efficient microbiome analysis software to scan the draft assembly of domestic cow, Bos taurus, and identify 173 small contigs that appeared to derive from microbial contaminants. In the course of verifying these findings, we discovered that one genome, Neisseria gonorrhoeae TCDCNG08107, although putatively a complete genome, contained multiple sequences that actually derived from the cow and sheep genomes. Our findings illustrate the need to carefully validate findings of anomalous DNA that rely on comparisons to either draft or finished genomes. 


\section{Unexpected cross-species contamination in genome sequencing projects}

2 Samier Merchant ${ }^{1,2}$, Derrick E. Wood ${ }^{1,3}$, and Steven L. Salzberg ${ }^{1,3,4,5}$

$3{ }^{1}$ Center for Computational Biology, McKusick-Nathans Institute of Genetic Medicine, Johns Hopkins University,

4 Baltimore, Maryland, U.S.A.

$5 \quad{ }^{2}$ Department of Computer Science, Brown University, Providence, Rhode Island, U.S.A.

$6 \quad{ }^{3}$ Department of Computer Science, Johns Hopkins University

$7 \quad{ }^{4}$ Department of Biomedical Engineering, Johns Hopkins University

$8 \quad{ }^{5}$ Corresponding author. Email: salzberg@jhu.edu. Address: Johns Hopkins School of Medicine, Welch Medical

9 Library, Rm 107, 1900 E. Monument St., Baltimore, MD 21205.

\section{Introduction}

11 Genome sequencing projects have dramatically increased in number and complexity in recent

12 years. The first complete bacterial genome, Haemophilus influenzae, appeared in 1995, and today

13 the public GenBank database contains over 27,000 prokaryotic and 1,600 eukaryotic genomes.

14 Although many of these are draft genomes that contain gaps in their sequences, over 3000 of the

15 prokaryotic genomes are listed as complete, meaning that every nucleotide is present with no

16 gaps.

17 The recent dramatic growth in microbiome research has been driven not only by the falling cost

18 of sequencing, but by this large and growing set of known genomes. The large set of completed

19 genomes makes it possible to identify, usually with high confidence, the species present in a

20 sample of DNA taken from a site on the human body. The accuracy of microbiome analysis is

21 critically dependent on the accuracy of the previously-sequenced microbial genomes. The vast

22 majority of these sequences are accurate, but any errors may be amplified by efforts to search for

23 the presence of unusual or unexpected species. This paper describes the finding of unexpected

24 contaminants in two published genomes and the methods used to identify them.

25 Each genome sequencing project begins with a DNA source, which varies depending on the

26 species. For animals, blood is a common source, while for smaller organisms such as insects the

27 entire organism or a population of organisms may be required to yield enough DNA for

28 sequencing. Throughout the process of DNA isolation and sequencing, contamination remains a

29 possibility. Computational filters applied to the raw sequencing reads are usually effective at

30 removing common laboratory contaminants such as E. coli, but other contaminants may be more

31 difficult to identify. Human DNA is another common contaminant, presumably from the

32 scientists who handle the samples at various times during the process of extraction through

33 sequencing (Longo et al. 2011).

34 The current project was initiated when we learned that a microbiome project studying samples

35 collected from domestic cows (Bos taurus) had identified the presence of a possible human

36 pathogen that does not infect cows. As we investigated further, we discovered, first, that some of

37 the original Bos taurus sequences were actually bacteria, and second, that some sequences from a

38 published genome of Neisseria gonorrhoeae were actually cow and sheep DNA.

\section{Methods}

40 We began by using microbiome sequence analysis software to analyze the genome of the

41 domestic cow, Bos taurus, for signs of microbial contamination. The Bos taurus genome was 
42 originally assembled from 35 million Sanger reads (Zimin et al. 2009). The vast majority of the

43 assembly (version UMD 3.1) was mapped onto chromosomes, but a small fraction remained

44 unmapped, as is common with all draft genomes. When we began our investigation, the UMD 3.1

45 assembly had 3,286 unmapped contigs containing 9,499,556 nucleotides.

46 To analyze the unplaced contigs from the Bos taurus genome, we used the Kraken system (Wood

47 and Salzberg 2014) to classify each contig. Kraken is a very fast method for identifying the

48 species represented by a DNA sequence, using exact matching of short subsequences of length $k$,

49 called $k$-mers. The software uses a specialized database of $k$-mers (where $k=31$ by default) that

50 can be constructed from any set of genomes. For our study, we built a database containing all

51 bacteria, archaea, and viruses. To classify a new sequence $S$, Kraken looks up every $k$-mer in $S$ to

52 determine if it exists in any known species. If a $k$-mer occurs in more than one species, Kraken

53 assigns it to the lowest common ancestor (LCA) of those species. After looking up every $k$-mer,

54 Kraken then uses a weighted voting scheme to determine the species or higher-order clade

55 assignment for $S$.

56 Our Kraken database contained 2,757 bacteria and archaeal genomes and 2,335 viral genomes

57 from the RefSeq database at NCBI (Tatusova et al. 2014). The Kraken software

58 (http://ccb.jhu.edu/software/kraken/) includes an automated program that will download all these

59 genomes directly from NCBI and build a local database. It also includes instructions on how to

60 build a database using a customized set of species.

61 After using Kraken to process the 3286 unmapped Bos taurus contigs, we ran a second analysis

62 looking at the protein translations of these contigs. For this analysis, we created a database with

63 all protein sequences from the 2,757 complete microbial genomes and used BLASTX (Camacho

64 et al. 2009) to align each contig to the database. As a quality control step, we also ran Kraken on

65 most of the mapped contigs, using all sequences from chromosomes 1 through 10 . All

66 experiments were run on a computer with $256 \mathrm{~GB}$ of RAM and four 2.1 GHz, 12-core AMD

67 Opteron processors. Kraken processed the 3286 unplaced contigs (9.5 megabases) in just 3.98

68 seconds.

\section{Results and Discussion}

70 After removing low-complexity contigs (some of which contained nothing other than a series of

71 dinucleotide repeats), 138 contigs from the Bos taurus UMD 3.1 assembly were identified as

72 bacterial in origin. The BLASTX search, which was far slower but more sensitive, confirmed

73 these 138 and identified 35 additional contaminants including both bacteria and viruses, for a

74 total of 173 contaminant contigs. Supplementary Table S1 lists all the contigs with the closest

75 matching microbial species for each one. The most common contaminants found belonged to the

76 genera Acinetobacter (29 contigs), Pseudomonas (35 contigs), and Stenotrophomonas (27

77 contigs). Note that additional microbial species might still be present but undetectable, if they

78 derive from organisms that are not similar to any sequenced species.

79 One interesting finding from the unplaced contigs was Bovine herpesvirus 6, isolate Pennsylvania

8047 , a cattle-specific virus that causes multiple diseases. Because this is a retrovirus, we

81 considered the possibility that it had actually inserted itself into the host genome - i.e., that it was 
82 part of the genome and not a contaminant - in which case we would expect parts of the sequence

83 to appear in the chromosomal contigs.

84 To evaluate this hypothesis, we used the nucmer program from the MUMmer package (Delcher

85 et al. 2002; Kurtz et al. 2004) to align the entire bovine herpesvirus genome against the entire

86 Bos taurus assembly. This alignment yielded the same five contigs (Supplementary Table S1,

87 contigs 149-153) we had found in our original scan, indicating that the virus was not integrated

88 into the chromosomal DNA but rather an infection in the original animal.

89 To reflect these findings, we created a new release of the Bos taurus assembly, numbered 3.1.1,

90 available as Bos_taurus_UMD_3.1.1 at NCBI (Accession GCF_000003055.5) and also available

91 from ccb.jhu.edu/bos_taurus_assembly.shtml.

92 We then used Kraken to search all of the sequences placed on chromosomes 1 through 10, as a

93 quality check on our method. We did not expect any of these contigs to match bacteria, but we

94 unexpectedly found 2,885 small contigs that seemed to align in part to a single bacterial genome,

95 Neisseria gonorrhoeae, strain TCDC-NG08107 (Chen et al. 2011). This bacterium is a human-

96 specific pathogen, and it seemed highly unlikely that it had contaminated the original DNA used

97 for sequencing.

98 Upon further investigation, we found that every contig aligned to one of just four locations on the 99 TCDC-NG08107 strain, shown in Table 1. The aligned regions ranged in length from 200 to 634

100 bp. When we extracted these sequences and aligned them separately to all sequences in

101 GenBank, all of the matching sequences were from Bos taurus.

102 In an effort to determine the source of these foreign sequences in the TCDC-NG08107 genome

103 (Genbank accession CP002440), we examined the original publication (Chen et al. 2011) and the

104 GenBank entry, and found that although the genome was listed as complete in GenBank, Chen et

105 al. described an assembly that comprised 180 contigs. Neither the publication nor the GenBank

106 entry contained any information that the gaps had been filled. We concluded that sequence was

107 erroneously uploaded as a finished genome, with all contigs simply concatenated together, and

108 that the cow and sheep sequences represented accidental contaminants, presumably inserted

109 computationally.

110 We then used the nucmer program (Kurtz et al. 2004) to align TCDC-NG08107 to its two closest

111 relatives among the complete bacterial genomes, strains FA1090 and NCCP11945 (GenBank

112 accessions AE004960 and CP001050), which were also used by Chen et al. to order and orient

113 their original set of 180 contigs. These alignments indicated 181 separate alignments, in close

114 agreement with the publication. We also found 67 small segments that did not align to either of

115 the related strains. Normally, these would represent sequences that are insertions in TCDC-

116 NG08107 as compared to other strains, a common finding when comparing bacterial genomes.

117 However, these small segments included the regions that had matched the cow genome (Table 1).

118 As a further check, we aligned all 67 segments to the NCBI comprehensive nucleotide database.

119 As shown in Table 1, four of these segments matched Bos taurus, and a fifth segment aligned to

120 Ovis aries (sheep). Not surprisingly, none of these five mammalian DNA fragments matched any

121 other microbial species. 
122 After removing the contaminated contigs, we used our alignments to re-order the remaining 123 contigs using both NCCP11945 and FA1090. We removed 11 contigs that were fully contained 124 within other contigs. This process yielded a reconstructed draft genome of TCDC-NG08107 with 125 a total of 165 contigs, available in the Supplementary Data. However, because we did not have 126 access to the original TCDC-NG08107 data and because the original submitters did not respond 127 to any requests for data, we cannot be confident that these contigs are the best representation of 128 the genome. As a result of our findings, GenBank has temporarily suppressed the entry for this

129 genome.

130 Contaminants in other genomes. As a test of whether these findings might apply to other publicly 131 available genomes, we randomly selected eight additional genomes from the NCBI database and 132 ran Kraken on each of them. The eight genomes range in size from 75-700 Mbp and include 133 animals, plants, and fungi. We also performed BLAST searches for each of the sequences that 134 Kraken identified as contaminants (Table 2), all of which were confirmed as microbial species. 135 Three of the eight genome assemblies contained just 2-4 contaminant contigs, and one $(C$.

136 reinhardtii) had 227, roughly similar to the number we found in Bos taurus.

\section{Conclusion}

138 These results illustrate the importance of performing a thorough search for contamination before

139 submitting a genome sequence to a public archive. The rapidly growing number of draft genomes

140 represents both a valuable resource and also, as we show here, a cautionary tale. Perhaps most

141 problematic was the presence of foreign DNA in N. gonorrhoeae TCDC-NG08107, a genome

142 that was submitted to GenBank as complete. If scientists cannot assume that the sequence of a

143 species truly comes from that species, then analyses that use this data may be fundamentally

144 flawed. Contamination from other species may masquerade as lateral gene transfer (Willerslev et

145 al. 2002), an event that is relatively common between some bacteria but extremely rare

146 otherwise. In particular, the transfer of bacterial DNA directly into a mammalian genome has

147 been suggested previously, based on compositional analysis, but never proven (Salzberg et al.

148 2001). The presence of erroneously labelled DNA causes particular problems for microbiome

149 analysis, in which the primary goal is the identification of which species are present in a sample.

150 These findings highlight the importance of careful screening of DNA sequence data both at the

151 time of release and, in some cases, for many years after publication.

\section{Supplementary data files}

153 Supplementary Table S1 contains the 173 putative microbial contigs that were removed from the 154 draft Bos taurus genome. The supplementary data also include two DNA sequence files. One file 155 contains the 165 re-ordered contigs that comprise the reconstruction of the $N$. gonorrhoeae 156 genome, with the contaminating sequences removed. The 5 contaminants are included as a 157 separate file.

\section{References}

159 Camacho, C., G. Coulouris, V. Avagyan, N. Ma, J. Papadopoulos, K. Bealer and T.L. Madden 160 (2009). "BLAST+: architecture and applications." BMC Bioinformatics 10: 421.

161 Chen, C.C., K.C. Hsia, C.T. Huang, W.W. Wong, M.Y. Yen, L.H. Li, K.Y. Lin, K.W. Chen and 162 S.Y. Li (2011). "Draft genome sequence of a dominant, multidrug-resistant Neisseria 

gonorrhoeae strain, TCDC-NG08107, from a sexual group at high risk of acquiring human immunodeficiency virus infection and syphilis." J Bacteriology 193(7): 17881789.

Delcher, A.L., A. Phillippy, J. Carlton and S.L. Salzberg (2002). "Fast algorithms for large-scale genome alignment and comparison." Nucleic Acids Research 30(11): 2478-2483.

Kurtz, S., A. Phillippy, A.L. Delcher, M. Smoot, M. Shumway, C. Antonescu and S.L. Salzberg (2004). "Versatile and open software for comparing large genomes." Genome Biology $\mathbf{5}(2): \mathrm{R} 12$.

Longo, M.S., M.J. O'Neill and R.J. O'Neill (2011). "Abundant human DNA contamination identified in non-primate genome databases." PLoS One 6(2): e16410.

Salzberg, S.L., O. White, J. Peterson and J.A. Eisen (2001). "Microbial genes in the human genome: lateral transfer or gene loss?" Science 292(5523): 1903-1906.

Tatusova, T., S. Ciufo, B. Fedorov, K. O'Neill and I. Tolstoy (2014). "RefSeq microbial genomes database: new representation and annotation strategy." Nucleic Acids Research 42(Database issue): D553-559.

Willerslev, E., T. Mourier, A.J. Hansen, B. Christensen, I. Barnes and S.L. Salzberg (2002). "Contamination in the draft of the human genome masquerades as lateral gene transfer." DNA Seq 13(2): 75-76.

Wood, D.E. and S.L. Salzberg (2014). "Kraken: ultrafast metagenomic sequence classification using exact alignments." Genome Biology 15(3): R46.

Zimin, A.V., A.L. Delcher, L. Florea, D.R. Kelley, M.C. Schatz, D. Puiu, F. Hanrahan, G. Pertea, C.P. Van Tassell, T.S. Sonstegard, G. Marcais, M. Roberts, P. Subramanian, J.A. Yorke and S.L. Salzberg (2009). "A whole-genome assembly of the domestic cow, Bos taurus." Genome Biology 10(4): R42. 
$\underline{\text { Table captions }}$

188 Table 1. Locations of foreign DNA in Neisseria gonorrhoeae TCDC-NG08107 genome. E189 values in column 4 were computed by the BLAST program in a search against the NCBI 190 comprehensive sequence database.

191 Table 2. Results of screening 8 publicly available draft genomes for microbial contaminants.

192 GenBank accession numbers are shown for each genome along with the number of contigs and 193 the size of the draft assembly. The last column shows the sequencing technology used for each 194 project.

195 Supplementary Table S1. 173 contigs from the Bos taurus assembly identified as possible 196 contaminants. The closest matching bacterial, archaeal, or viral species is shown. Sequence ID 197 refers to the original identifier in the Bos taurus UMD 3.1 assembly. All contigs belonged to the 198 unmapped set; none were mapped onto chromosomes. The final column shows the BLAST E199 value from an alignment of each contig against the comprehensive DNA sequence database "nr" 200 at NCBI. 


\section{Table $\mathbf{1}$ (on next page)}

Table 1

Locations of foreign DNA in Neisseria gonorrhoeae TCDC-NG08107 genome. E-values in column 4 were computed by the BLAST program in a search against the NCBI comprehensive sequence database. 
Table 1. Locations of foreign DNA in Neisseria gonorrhoeae TCDC-NG08107 genome. E-values in column 4 were computed by the BLAST program in a search against the NCBI comprehensive sequence database.

\begin{tabular}{|l|l|l|l|}
\hline $\begin{array}{l}\text { Genome } \\
\text { coordinates }\end{array}$ & Length & $\begin{array}{l}\text { True } \\
\text { species }\end{array}$ & BLAST E-Value \\
\hline $499351-499709$ & 359 & Cow & $3 \times 10^{-168}$ \\
\hline $1267185-1267393$ & 209 & Cow & $1 \times 10^{-71}$ \\
\hline $1371560-1371932$ & 373 & Cow & $2 \times 10^{-130}$ \\
\hline $1635755-1635954$ & 200 & Cow & $3 \times 10^{-93}$ \\
\hline $2118014-2118647$ & 634 & Sheep & 0.0 \\
\hline
\end{tabular}




\section{Table 2 (on next page)}

Table 2

Results of screening 8 publicly available draft genomes for microbial contaminants. GenBank accession numbers are shown for each genome along with the number of contigs and the size of the draft assembly. The last column shows the sequencing technology used for each project. 
Table 2. Results of screening 8 publicly available draft genomes for microbial contaminants. GenBank accession numbers are shown for each genome along with the number of contigs and the size of the draft assembly. The last column shows the sequencing technology used for each project.

\begin{tabular}{|c|c|c|c|c|c|c|}
\hline Genome & $\begin{array}{c}\text { \# of } \\
\text { contaminant } \\
\text { contigs }\end{array}$ & $\begin{array}{c}\text { Total } \\
\text { contaminant } \\
\text { length (bp) }\end{array}$ & $\begin{array}{l}\text { Range of E- } \\
\text { values }\end{array}$ & $\begin{array}{l}\text { Total \# of } \\
\text { contigs }\end{array}$ & $\begin{array}{l}\text { Genome } \\
\text { size } \\
(\mathrm{Mbp})\end{array}$ & Technology \\
\hline $\begin{array}{l}\text { Schistosoma } \\
\text { haematobium } \\
\text { (GCA_000699445.1) }\end{array}$ & 4 & 9,415 & $0.0-4 \mathrm{E}-71$ & 49,195 & 35 & Illumina \\
\hline $\begin{array}{l}\text { Cynoglossus } \\
\text { semilaevis } \\
\text { (GCA_000523025.1) }\end{array}$ & 2 & 904 & $1 \mathrm{E}-6-5 \mathrm{E}-22$ & 62,912 & 470 & Illumina \\
\hline $\begin{array}{l}\text { Caenorhabditis } \\
\text { brenneri } \\
\text { (GCA_000143925.2) }\end{array}$ & 2 & 19,677 & 0.0 & 13,373 & 190 & $\begin{array}{l}\text { ABI Solid } \\
\text { Sequencing }\end{array}$ \\
\hline $\begin{array}{l}\text { Chlamydomonas } \\
\text { reinhardtii } \\
\text { (GCA_000002595.2) }\end{array}$ & 227 & 254,869 & 0.0 & 11,385 & 120 & Sanger \\
\hline $\begin{array}{l}\text { Citrus clementina } \\
\text { (GCA_000493195.1) }\end{array}$ & 0 & 0 & N/A & 8,962 & 301 & Sanger \\
\hline $\begin{array}{l}\text { Anopheles darlingi } \\
\text { (GCA_000211455.3) }\end{array}$ & 0 & 0 & N/A & 13,857 & 174 & 454 \\
\hline $\begin{array}{l}\text { Auricularia delicata } \\
\text { TFB-10046 SS5 } \\
\text { (GCA_000265015.1) }\end{array}$ & 0 & 0 & N/A & 4,884 & 75 & Illumina \\
\hline $\begin{array}{l}\text { Schmidtea } \\
\text { mediterranea } \\
\text { (GCA_000691995.1) }\end{array}$ & 0 & 0 & N/A & 118,433 & 701 & Illumina \\
\hline
\end{tabular}

